\title{
Observation of non-Hermitian degeneracies in a chaotic exciton-polariton billiard
}

T. Gao ${ }^{1}$, E. Estrecho ${ }^{1}$, K.Y. Bliokh ${ }^{1,2}$, T.C.H. Liew ${ }^{3}$, M.D. Fraser ${ }^{2}$, S. Brodbeck ${ }^{4}$, M. Kamp ${ }^{4}$, C. Schneider ${ }^{4}$, S. Höfling ${ }^{4,5}$, Y. Yamamoto ${ }^{6,7}$, F. Nori ${ }^{2,8}$, Y.S. Kivshar ${ }^{1}$, A.G. Truscott ${ }^{1}$, R.G. Dall ${ }^{1}$, and E.A. Ostrovskaya ${ }^{1}$

${ }^{1}$ Research School of Physics and Engineering, The Australian National University, Canberra, ACT 2601, Australia

${ }^{2}$ Center for Emergent Matter Science, RIKEN, Wako-shi, Saitama 351-0198, Japan

${ }^{3}$ School of Physical and Mathematical Sciences, Nanyang Technological University, 637371, Singapore

${ }^{4}$ Technische Physik and Wilhelm-Conrad-Röntgen Research Center for Complex Material Systems, Universität Würzburg, Am Hubland, D-97074 Würzburg, Germany

${ }^{5}$ SUPA, School of Physics and Astronomy, University of St Andrews, St Andrews KY16 9SS, United Kingdom

${ }^{6}$ ImPACT Project, Japan Science and Technology Agency, Chiyoda-ku, Tokyo 102-0076, Japan

${ }^{7}$ Edward L. Ginzton Laboratory, Stanford University, Stanford, California 94305-4085, USA

${ }^{8}$ Physics Department, University of Michigan, Ann Arbor, Michigan 48109-1040, USA

Exciton-polaritons are hybrid light-matter quasiparticles formed by strongly interacting photons and excitons (electron-hole pairs) in semiconductor microcavities ${ }^{1-3}$. They have emerged as a robust solid-state platform for next-generation optoelectronic applications as well as fundamental studies of quantum many-body physics. Importantly, excitonpolaritons are a profoundly open (i.e., non-Hermitian ${ }^{4,5}$ ) quantum system: it requires constant pumping of energy and continuously decays releasing coherent radiation ${ }^{6}$. Thus, the exciton-polaritons always exist in a balanced potential landscape of gain and loss. However, the inherent non-Hermitian nature of this potential has so far been largely ignored in exciton-polariton physics. Here we demonstrate that non-Hermiticity dramatically modifies the structure of modes and spectral degeneracies in excitonpolariton systems, and, therefore, will affect their quantum transport, localisation, and dynamical properties $^{7-9}$. Using a spatially-structured optical pump ${ }^{10-12}$, we create a chaotic exciton-polariton billiard. Eigenmodes of this billiard exhibit multiple non-Hermitian spectral degeneracies - exceptional points ${ }^{13,14}$. These are known to cause remarkable wave phenomena, such as unidirectional transport ${ }^{15}$, anomalous lasing/absorption ${ }^{16,17}$, and chiral modes $^{18}$. By varying parameters of the billiard, we observe crossing and anti-crossing of energy levels and reveal the nontrivial topological modal structure exclusive to nonHermitian systems ${ }^{9,13-22}$. We also observe the mode switching and topological Berry phase for a parameter loop encircling the exceptional point ${ }^{23,24}$. Our findings pave the way for studies of non-Hermitian quantum dynamics of exciton-polaritons, which can uncover novel operating principles for polariton-based devices.

Studies of open quantum systems go back to Gamow's theory of nuclear alpha-decay developed in the early days of quantum mechanics ${ }^{4}$. Indeed, metastable states of a single quantum particle in a spherically symmetric potential well with semi-transparent barriers decay in time, and therefore are characterized by complex energies. Furthermore, introducing a 2D potential well with nontrivial geometry, i.e., quantum billiard, results in strongly correlated energy levels and transition to quantum chaos ${ }^{7,19,21,25-28}$. Spectral degeneracies crucially determine transport and dynamical properties in both non-Hermitian and chaotic wave systems ${ }^{{ }^{7-}}$ 9,15-17. In chaotic and disordered wave systems, spectral degeneracies underpin statistical properties and quantum phase transitions from the localised to delocalised dynamics ${ }^{8,9}$. In non- 
Hermitian (including $\mathcal{P} \mathcal{T}$-symmetric) systems, nontrivial topology of eigenmodes and unusual transport properties in the vicinity of exceptional points ${ }^{15-17,19}$ are currently under intense investigation. To date, basic non-Hermitian or stochastic dynamics were studied in the context of microwave ${ }^{9,18-20,24}$, optical ${ }^{15-17,19,21}$, atomic ${ }^{22,26,27}$, and electron ${ }^{25,28}$ waves. However, the concepts of non-Hermiticity and quantum chaos remain largely separated from each other, due to the lack of a simple quantum system in which both features would be readily accessible. Moreover, it is challenging to produce artificial complex potentials with gain and loss for classical waves, as well as to observe nanoscopic electron states in solids.

Microcavity exciton-polaritons represent a unique quantum macroscopic system, which combines the main advantages of light and matter waves ${ }^{1-3}$. Being bosons, exciton-polaritons can display collective quantum behaviour, Bose-Einstein condensation (BEC), when they occupy a single-particle quantum state in massive numbers. Exciton-polaritons have provided a very accessible playground for studies of the collective quantum behaviour because they condense at the temperatures ranging from $10 \mathrm{~K}$ to room temperature (compared to nano-Kelvins for neutral atoms) and do not require painstaking isolation from the environment.

The schematics of exciton-polariton condensation under continuous-wave incoherent optical excitation conditions ${ }^{1}$ are shown in Fig. 1a. The optical pump, far detuned from the exciton resonance in the cavity, effectively creates an incoherent reservoir of 'hot', exciton-like polaritons. Above a threshold phase-space density of the reservoir, relaxation and stimulated scattering into the coherent BEC state of exciton-polaritons dominate the dynamics. The continuously pumped condensate decays releasing coherent photons, which escape the cavity carrying all information about the condensed state. The interactions between the reservoir and condensed exciton-polaritons are responsible for the formation of effective pump-induced potentials $^{10-12}$. Thus, the macroscopic matter wave function is shaped by an optical pump and spatially resolved via free-space optical microscopy. This enables us to clearly observe and control non-Hermitian and irregular quantum dynamics.

We use a structured optical pump ${ }^{10-12}$ to create a non-Hermitian potential in the shape of a Sinai billiard with a circular defect of radius $R^{7}$ (see Fig. 1b) for condensed exciton-polaritons (see Methods for details). In our experiment the billiard has 'soft' (inelastic) walls of a finite width and height. The main properties of eigenstates of the exciton-polariton condensate in the billiard can be described by a linear Schrodinger equation with a complex two-dimensional potential $V(\mathbf{r})=V^{\prime}(\mathbf{r})+i V^{\prime \prime}(\mathbf{r})$. Here $V^{\prime}(\mathbf{r}) \propto P(\mathbf{r})$ is the potential barrier shaped as a Sinai billiard boundary with a Gaussian envelope. This potential is proportional to the optical pump rate, $P(\mathbf{r})$, and is induced by the strong repulsive interaction between the excitonic reservoir populated by the pump and the polariton $\mathrm{BEC}^{10-12}$. The imaginary part of the potential, $V^{\prime \prime}(\mathbf{r}) \propto P(\mathbf{r})-\gamma$, combines the gain profile produced by the same optical pump $P(\mathbf{r})$ with the spatially-uniform loss $\gamma$ due to the polariton decay (Fig. 1b). Despite the strong polaritonpolariton interactions, the corresponding nonlinearity mostly affects the relative population of the energy eigenstates, as well as the overall blueshift (see Methods).

Changing the radius of the defect, $R$, varies the geometry of the billiard and hence affects the energy levels. Figures $1 \mathrm{c}$ and $1 \mathrm{~d}$ show the experimentally measured and numerically computed energy spectra $E(R)$ of the first 11 levels as a function of $R$. Variations of the shape of the 2D potential tunes eigenvalues of different modes at different rates, and as a result some energy levels approach each other at certain values of $R$. One can see (Fig. 1c,d) that multiple degeneracies (or near-degeneracies) appear in the spectrum. In a 'hard-wall' Hermitian Sinai billiard, the proliferation of degeneracies is a signature of the transition from regular to chaotic dynamics ${ }^{7}$. Although our exciton-polariton billiard has 'soft' walls and can generically exhibit mixed regular-chaotic behaviour ${ }^{27}$, we clearly observe multiple degeneracies similar to the 'hardwall' case ${ }^{7}$. In Hermitian billiards, the levels generically avoid crossing (anti-cross) in the vicinity of degeneracies, which correspond to the average level repulsion and Wigner distribution of the nearest-neighbour energy spacings ${ }^{8}$. In contrast, the non-Hermitian systems 
can exhibit both crossings and anti-crossings of levels ${ }^{9,19-22}$. This is because the energy eigenvalues in non-Hermitian systems are complex: the real part and imaginary parts correspond to the real energies and linewidths of the modes, respectively. A crossing of the energies is accompanied by an anti-crossing of the linewidths and vice versa. In our experiment, we measure the spectral profile of the cavity photoluminescence at a particular spatial position and extract both peak energies and widths of spectral resonances (see Methods). Crossings as well as anti-crossings of real energy levels are clearly seen both in experiments (Fig. 1b) and numerics (Fig. 1c).

To observe the transition between the crossing and anti-crossing for the same neardegenerate pair of eigenvalues, one needs to vary a second control parameter. In our excitonpolariton billiard this additional parameter is the thickness, $d$, of the billiard walls. Provided the internal area of the billiard remains intact, this parameter does not affect the geometry of the billiard and primarily controls the imaginary part $V^{\prime \prime}$ of the non-Hermitian potential barrier. Figure 2 shows one pair of billiard modes highlighted in Fig. $1 \mathrm{~b}$ in the vicinity of a neardegeneracy for two values of the control parameter $d$. One can clearly see the anti-crossing (crossing) behaviour of the real (imaginary) parts of the complex eigenenergies in the billiard with thick walls (Figs. 2a,c) and the opposite behaviour for the thin-wall billiard (Figs. 2b,d).

Importantly, the energy-resolved real-space imaging of the photoluminescence provides all the information about complex eigenvalues as well as the spatial structure of the eigenmodes (wave functions). In particular, the levels shown in Figure 2 correspond (at $R=0$ ) to the third mode with three horizontal lobes and the forth mode with two vertical lobes. The experimentally imaged and calculated spatial profiles of these eigenmodes are shown as insets in Figures $2 \mathrm{a}, \mathrm{b}$ along the eigenenergy curves. We observe that the two modes are hybridised and therefore change their spatial profiles in the near-degeneracy region, and 'exchange' their spatial profiles after passing it.

The behaviour of two billiard modes in the vicinity of a degeneracy can be described by a simple model of a two-level system with an effective coupling (see Methods). The corresponding non-Hermitian Hamiltonian reads ${ }^{9,18-22}$ :

$$
\hat{H}=\left(\begin{array}{cc}
\tilde{E}_{1} & q \\
q^{*} & \tilde{E}_{2}
\end{array}\right), \quad \tilde{E}_{1,2}=E_{1,2}-i \Gamma_{1,2} .
$$

Here $\tilde{E}_{1,2}$ are the complex eigenevalues of two uncoupled modes (with $E_{1,2}$ being the real energies and $\Gamma_{1,2}$ being the decay/gain rates), whereas $q$ characterizes the coupling between these two modes. We will also use the mean complex energy $\tilde{E}=\left(\tilde{E}_{1}+\tilde{E}_{2}\right) / 2 \equiv E-i \Gamma$, and the complex energy difference $\delta \tilde{E}=\left(\tilde{E}_{2}-\tilde{E}_{1}\right) / 2 \equiv \delta E-i \delta \Gamma$. The eigenvalues of the Hamiltonian (1) are $\lambda_{1,2}=\tilde{E} \pm \sqrt{\delta \tilde{E}^{2}+|q|^{2}}$; their real and imaginary parts, which depend on the parameters $\delta \tilde{E}=(\delta E, \delta \Gamma)$, are shown in Figure 3. These complex eigenvalues coalesce, $\lambda_{1}=\lambda_{2}$, at the exceptional points ${ }^{13-22}$, where $i \delta \tilde{E}_{\mathrm{EP}}= \pm|q|$. At these points, the eigenstates also coalesce and form a single chiral mode ${ }^{13,14,18}$. Assuming that the coupling constant $q$ is fixed, the exceptional points appear in the parameter plane as $\left(\delta E_{\mathrm{EP}}, \delta \Gamma_{\mathrm{EP}}\right)=(0, \pm|q|)$. We assume $\delta \Gamma>0$ in our range of parameters, so that there is only one exceptional point in the domain of interest. The exceptional point can be encircled in the $(\delta E, \delta \Gamma)$ plane by varying these two parameters, as seen in Fig. 3. 
Two parameters of the model, $(\delta E, \delta \Gamma)$, approximately correspond to the varying parameters $(R, d)$ of our exciton-polariton billiard. The radius $R$ mostly affects the real part of the potential, $V^{\prime}(\mathbf{r})$, and hence the energy difference between the modes. Increasing $R$ corresponds to a tighter spatial confinement and therefore to increasing $\delta E$. In turn, the thickness $d$ of the billiard walls controls the gain/loss profile $V^{\prime \prime}(\mathbf{r})$. Different modes have different spatial overlaps with the imaginary potential $V^{\prime \prime}(\mathbf{r})$, and, therefore, are characterised by different integral (spatially-averaged) dissipation parameters $\Gamma_{1,2}$ (see Methods). In our case, increasing $d$ corresponds to decreasing $\delta \Gamma$. The effective coupling $q$ in model (1) is determined by the spatial overlap between the two modes away from the hybridisation region? The red and blue curves in Figure 3 show the crossing/anti-crossing behaviour of the real and imaginary parts of the eigenvalues versus the energy difference $\delta E$ for two values of the dissipation parameter: $\delta \Gamma<\delta \Gamma_{\mathrm{EP}}$ and $\delta \Gamma>\delta \Gamma_{\mathrm{EP}}$. This behaviour is perfectly consistent with that in the experimental Figure 2, which means that our range of varying parameters includes the exceptional point.

The complex-eigenvalues structure in the vicinity of the exceptional point reveals nontrivial topology of a branch-point type ${ }^{13-22}$ shown in Figure 3. Therefore, continuous encircling of the non-Hermitian degeneracy in the two-parameter plane (e.g., along the green contour in Fig. 3) results in the transition to the other branch. When the contour is traversed twice, we return to the original mode, most significantly with a $\pi$ topological phase shift ${ }^{23,24}$. We use the method suggested in the microwave experiment ${ }^{24}$ to trace the above topological structure of two modes in the vicinity of the exceptional point. Namely, we compare the eigenmodes at neighbouring values of parameters $(\delta E, \delta \Gamma) \sim(R, d)$ along the contour encircling the exceptional point (see Fig. 3). Notably, we do not consider adiabatic evolution of modes due to variations of the parameters $(R, d)$ in time; such evolution would be accompanied by unavoidable nonadiabatic transitions in the non-Hermitian case ${ }^{29,30}$. Rather, we examine the natural topological structure and geometrical connection of stationary modes depending on the parameter values. Figure 4 depicts the experimentally measured intensities and the corresponding numerically simulated phase profiles of the two modes from Fig. 2 for the parameters values lying on the contour encircling the exceptional point (Fig. 3). In Figure 4a, we start on the upper branch (blue in Figs. 2a and 3a) at $R<R_{\mathrm{EP}}, d>d_{\mathrm{EP}}$ and trace the eigenmode transmutation as the radius is increased to $R>R_{\mathrm{EP}}$. This takes us from the vertical two-lobe mode, through the anti-crossing, to the horizontal three-lobe mode (still on the blue upper branch). Then, we decrease the thickness to $d<d_{\mathrm{EP}}$ and stay on the same horizontal three-lobe mode, which now corresponds to the red branch in Figs. $2 \mathrm{~b}$ and $3 \mathrm{a}$. Next, reducing the radius $R$ takes this mode through the crossing and recovers its three-lobe structure. Increasing $d$ closes the loop. Thus, the continuous transformation brought us from the vertical two-lobe mode ('start' in Figure 4a) to the horizontal three-lobe mode ('end' in Figure 4a) at the same values of parameters. Repeating this traverse one more time (Figure $4 \mathrm{~b}$ ) returns us to the original vertical two-lobe mode, but now with the $\pi$ topological phase shift (clearly seen in the simulated phase profiles). The experimental density distribution of the modes is in very good agreement with that calculated numerically. Therefore we can associate the phase structure of the simulated spatial modes with the experimental mode profiles ${ }^{24}$.

Thus, we have demonstrated creation of highly controllable complex (non-Hermitian) potentials for exciton-polaritons, and implemented a chaotic non-Hermitian exciton-polariton billiard with multiple spectral degeneracies. We have provided detailed experimental observations of the non-trivial behaviour of complex eigenvalues and eigenmodes in the vicinity of an exceptional point. These include crossing/anti-crossing transitions as well as mode switching and topological Berry phase when encircling the exceptional point in the two- 
parameter plane. Our results show that the inherent non-Hermitian nature of exciton-polaritons determines their basic properties, which are crucial for transport and quantum information processing. Therefore, these features should be taken into account in future studies and applications involving confinement and manipulation of exciton-polaritons. Most importantly, this complex quantum dynamics can bring novel functionality to polariton-based devices operating at the interface between photonics and electronics. Generally, exciton-polaritons offer a novel macroscopic quantum platform for studies of non-Hermitian physics and quantum chaos at the confluence of light and matter.

\section{References}

1. Kasprzak, J. et al. Bose-Einstein condensation of exciton polaritons, Nature 443, 409-414 (2006)

2. Deng, H., Haug, H. \& Yamamoto, Y. Exciton-polariton Bose-Einstein condensation, Rev. Mod. Phys. 82, 1489 (2010).

3. Carusotto, I. \& Ciuti, C. Quantum fluids of light, Rev. Mod. Phys. 85, 299 (2013).

4. Moiseyev, N. Non-Hermitian Quantum Mechanics (Cambridge University Press, 2011).

5. Bender, C. M. Making sense of non-Hermitian Hamiltonians, Rep. Prog. Phys. 70, 9471018 (2007).

6. Savvidis, P. G. et al. Off-branch polaritons and multiple scattering in semiconductor microcavities, Phys. Rev. B 64, 075311 (2001).

7. Berry, M. V. Quantizing a classically ergodic system: Sinai's billiard and the KKR method, Ann. Phys. 131, 163-216 (1981).

8. Guhr, T., Müller-Groeling, A. \& Weidenmüller, H. A. Random-matrix theories in quantum physics: common concepts, Phys. Rep. 299, 189-425 (1998).

9. Bliokh, K. Y., Bliokh, Y. P., Freilikher, V., Genack, A. Z. \& Sebbah, P. Coupling and level repulsion in the localized regime: From isolated to quasiextended modes, Phys. Rev. Lett. 101, 133901 (2008).

10. G. Tosi, G. Christmann, N. G. Berloff, P. Tsotsis, T. Gao, Z. Hatzopoulos, P. G. Savvidis, and J. J. Baumberg, Sculpting oscillators with light within a nonlinear quantum fluid, Nature Phys. 8, 190-194 (2012)

11. Sanvitto, D. et al. All-optical control of the quantum flow of a polariton condensate, Nature Photon. 5, 610-614 (2011).

12. Dall, R. et al. Creation of orbital angular momentum states with chiral polaritonic lenses, Phys. Rev. Lett. 113, 200404 (2014).

13. Berry, M. V. Physics of non-Hermitian degeneracies, Czech. J. Phys. 54, 1039-1047 (2004).

14. Heiss, W. D. The physics of exceptional points, J. Phys. A: Math. Gen. 45, 444016 (2012).

15. Guo, A. et al. Observation of $\mathcal{P} \mathcal{T}$-symmetry breaking in complex optical potentials, Phys. Rev. Lett. 103, 093902 (2009).

16. Peng, B. et al. Loss-induced suppression and revival of lasing, Science 346, 328-332 (2014).

17. Sun, Y., Tan, W., Li, H.-Q., Li, J. \& Chen, H. Experimental demonstration of a coherent perfect absorber with $\mathcal{P} \mathcal{T}$ phase transition, Phys. Rev. Lett. 112, 143903 (2014).

18. Dembowski, C. et al. Observation of a Chiral State in a Microwave Cavity, Phys. Rev. Lett. 90, 034101 (2003).

19. Cao, H. \& Wiersig, J. Dielectric microcavities: Model systems for wave chaos and nonHermitian physics, Rev. Mod. Phys. 87, 61-111 (2015).

20. Dembowski, C. et al. Experimental observation of the topological structure of exceptional points, Phys. Rev. Lett. 86, 787-790 (2001).

21. Lee, S.-B. et al. Observation of an exceptional point in a chaotic optical microcavity, Phys. Rev. Lett. 103, 134101 (2009). 
22. Choi, Y. et al. Quasi-eigenstate coalescence in an atomic-cavity quantum composite, Phys. Rev. Lett. 104, 153601 (2010).

23. Heiss, W. D. Phases of wave functions and level repulsion, Eur. Phys. J. D 7, 1-4 (1999).

24. Dembowski, C. et al. Encircling an exceptional point, Phys. Rev. E 69, 056216 (2004).

25. Akis, R., Ferry, D. K. \& Bird, J. P. Wave function scarring effects in open stadium shaped quantum dots, Phys. Rev. Lett. 79, 123-126 (1997).

26. Milner, V., Hanssen, J. L., Campbell, W. C. \& Raizen, M. G. Optical billiards for atoms, Phys. Rev. Lett. 86, 1514-1517 (2001).

27. Kaplan, A., Friedman, N., Anderson, M. \& Davidson, N. Observation of islands of stability in soft wall atom-optics billiards, Phys. Rev. Lett. 87, 274101 (2001).

28. Ponomarenko, L.A. et al. Chaotic Dirac billiard in graphene quantum dots, Science 320, 356-358 (2008).

29. Uzdin, R., Mailybaev, A., \& Moiseyev, N. On the observability and asymmetry of adiabatic state flips generated by exceptional points, J. Phys. A: Math. Theor. 44, 435302 (2011).

30. Berry, M. V. \& Uzdin, R. Slow non-Hermitian cycling: exact solutions and the Stokes phenomenon, J. Phys. A: Math. Theor. 44, 435303 (2011).

Acknowledgements We are grateful to Prof. Michael Berry and Dr. Oleg Kirillov for insightful comments. This research was supported by the Australian Research Council, the ImPACT Program of Council for Science, Technology and Innovation (Cabinet Office, Government of Japan), RIKEN iTHES Project, MURI Center for Dynamic Magneto-Optics, Grant-in-Aid for Scientific Research (A), and the State of Bavaria.

Author Contributions E.A.O., K.Y.B., T.G., and E.E. concieved the research; T.G., E.E., M.D.F., R.G.D. and A.G.T. designed and built the experiment; T.G., E.E., and R.G.D. collected and analysed experimental data; K.Y.B, E.E., T.C.H.L, and E.A.O. performed theoretical and numerical analysis; S.B., M.K., C.S., and S.H. fabricated and calibrated the semiconductor microcavity; E.A.O. and K.Y.B. wrote the paper with input from T.G., E.E., and T.C.H.L.; F.N., M.D.F., A.G.T, S.H., Y.Y., and Y.S.K. contributed to discussions and shaping of the manuscript.

Author Information Reprints and permissions information is available at www.nature.com/reprints. The authors declare no competing financial interests. Correspondence and requests for materials should be addressed to E.A.O. (elena.ostrovskaya@anu.edu.au). 
Figure 1. Non-Hermitian exciton-polariton Sinai billiard and its spectrum. (a) Excitonpolariton dispersion showing the incoherent excitonic reservoir, continuously replenished by the optical pump and polariton BEC decaying into cavity photoluminescence ${ }^{1-3}$. (b) Schematics of the exciton-polariton Sinai billiard formed in the plane of a quantum well embedded into the microcavity (see Methods). The barrier is induced by the optical pump via the excitonic reservoir, and the square modulus of the wave function of the confined polariton BEC (shown in greyscale inside the billiard) is imaged via the photoluminescence. The billiard dimensions are $W=14 \mu \mathrm{m}, L=23 \mu \mathrm{m}$, the radius of the defect $R$ is varied from 0 to $W$, and the thickness of the walls $d$ is varied from $3 \mu \mathrm{m}$ to $7 \mu \mathrm{m}$ (see Methods). Experimentally measured (c) and numerically simulated (d) spectra $E(R)$ for the first 11 modes of the billiard. With growing $R$, numerous degeneracies and quasi-degeneracies proliferate in the grey area, which is a signature of the transition to quantum chaos in the Hermitian Sinai billiards ${ }^{7}$. Topological properties of two near-degenerate modes (red and blue in the yellow rectangle) are analysed in detail in Figs. 2-4.

Figure 2. Crossing and anti-crossing for the two near-degenerate modes highlighted in Figs. 1c,d. Experimentally observed anti-crossing (a) and crossing (b) of eigenenergies of two modes in the spectrum of the exciton-polariton Sinai billiard with varying parameter $R$ (see Fig. 1) for thick, $d \simeq 6 \mu \mathrm{m},(\mathbf{a}, \mathbf{c})$ and thin, $d \simeq 4 \mu \mathrm{m},(\mathbf{b}, \mathbf{d})$ billiard walls; $d_{E P}$ is the value corresponding to the exceptional point. Panels (c) and (d) show the corresponding crossing and anti-crossing of the linewidths (i.e., imaginary parts of the complex eigenvalues). The upper (lower) inset panels in $(a, b)$ illustrate the numerically calculated (experimentally imaged) spatial structure of the eigenmodes at different values of the parameter $R$. Details of the hybridisation region are found in Methods.

Figure 3. Eigenvalues of a two-level non-Hermitian model in the vicinity of the exceptional point. Real (a) and imaginary (b) parts of the eigenvalues $\lambda_{1,2}$ of the model (1) as functions of two parameters $\delta E$ and $\delta \Gamma$. The exceptional point is shown in magenta. The crossing and anticrossing of the real and imaginary parts of the eigenvalues as functions of $\delta E$, for $\delta \Gamma<\delta \Gamma_{\mathrm{EP}}$ and $\delta \Gamma>\delta \Gamma_{\mathrm{EP}}$, are shown in red and blue. This is in correspondence with the experimentally observed behaviour in Fig. 2. Traversing along the green contour encircling the exceptional point in the $(\delta E, \delta \Gamma)$ plane reveals the nontrivial topology of eigenmodes, as shown in Fig. 4.

Figure 4. Observation of the topological Berry phase for circling around the exceptional point in the parameter plane. Transmutations of spatial distributions of the selected eigenmode (from the pair shown in Fig. 2) along the closed contour in the parameter space $(R, d) \sim(\delta E, \delta \Gamma)$ encircling the exceptional point (see Fig. 3). Parameters are not varied in time during the measurements, and each distribution corresponds to the stationary mode at the corresponding parameters values. The first loop (a) shows the transition to a different branch (mode) through the hybridization region (see explanations in text). The second loop (b) returns the mode to the original one with a $\pi$ topological phase shift $^{23,24}$. The phases are inferred from comparison with the numerically calculated modes. 